\title{
A Comonotonic Image of Independence for Additive Risk Measures
}

\author{
Marc J. Goovaerts ${ }^{\dagger, \ddagger}$, Rob Kaas ${ }^{\dagger}$, Roger J.A. Laeven ${ }^{\dagger, *}$, Qihe Tang ${ }^{\dagger}$ \\ ${ }^{\dagger}$ University of Amsterdam, Dept. of Quantitative Economics, Roetersstraat 11, \\ 1018 WB Amsterdam, The Netherlands \\ ${ }^{\ddagger}$ Catholic University of Leuven, Center for Risk and Insurance Studies, \\ Naamsestraat 69, B-3000 Leuven, Belgium
}

August 9, 2004

\begin{abstract}
This paper presents a new axiomatic characterization of risk measures that are additive for independent random variables. In contrast to previous work, we include an axiom that guarantees monotonicity of the risk measure. Furthermore, the axiom of additivity for independent random variables is related to an axiom of additivity for comonotonic random variables. The risk measure characterized can be regarded as a mixed exponential premium.
\end{abstract}

Keywords: Risk measures, Additivity, Exponential order, Laplace transform order, Esscher transform, Comonotonicity

JEL-Classification: D81, G22

*Corresponding author. E-mail: R.J.A.Laeven@uva.nl, Phone: +31 20525 7317, Fax: +31 205254349. 


\section{Introduction}

Several representations of risk measures that are additive for independent random variables are available in the literature. The most general representation has been characterized axiomatically by Gerber \& Goovaerts (1981) and is known as the mixed Esscher principle. More restrictive characterizations can be found in Gerber (1974) and Goovaerts \& De Vijlder (1980).

The mixed Esscher premium in general and the non-mixed Esscher premium in particular have several appealing features; the interested reader is referred to Bühlmann (1980), Gerber (1980) and Goovaerts, De Vijlder \& Haezendonck (1984). However, a serious concern of both the mixed and the non-mixed Esscher premium is that it is not monotonic in general, i.e., it does not in general preserve stochastic dominance; see Gerber (1981) and Van Heerwaarden, Kaas \& Goovaerts (1989).

In the present contribution we provide a new axiomatic characterization of risk measures that are additive for independent random variables. The characterization includes an axiom that guarantees monotonicity of the representing risk measure. Moreover, the current characterization relates the axiom of additivity of the risk measure for independent random variables to an axiom of additivity for comonotonic random variables. The risk measure obtained can be regarded as an ordinary mixture of exponential premiums. Equivalently, the obtained risk measure can be regarded as a restricted version of the mixed Esscher principle. In particular, the mixture function of the mixed Esscher principle is now required to be concave on $(0,+\infty)$ and convex on $(-\infty, 0)$, in addition to being non-decreasing.

\section{A New Axiomatic Representation of Additive Risk Measures}

In this section we present a new axiomatic characterization of risk measures that are additive for independent r.v.'s. Throughout the paper we restrict ourselves to bounded r.v.'s, unless stated otherwise. For a given r.v. $X$, we define the real-valued function $\varphi_{X}(\cdot)$ as follows:

$$
\varphi_{X}(t)= \begin{cases}\frac{1}{t} \log \mathbb{E}\left[e^{t X}\right], & t \neq 0 \\ \mathbb{E}[X], & t=0\end{cases}
$$

In the actuarial literature, $\varphi_{X}(t)$ for $t \geq 0$ is known as the exponential premium with parameter $t$, see Gerber (1974) and Goovaerts, De Vijlder \& Haezendonck (1984). For $t<0$, one may also regard the number $\varphi_{X}(t)$ as an exponential premium, but then it can 
be shown to have a negative safety loading. Notice that the correspondence between the cumulative distribution function (cdf) of $X$ and the function $\varphi_{X}(\cdot)$ is unique, since $\varphi_{X}(\cdot)$ corresponds uniquely to the moment generating function of $X$.

Next, for the cdf $F_{X}(\cdot)$ with differential $d F_{X}(\cdot)$, corresponding to a given r.v. $X$, we define by

$$
d F_{X}^{(t)}(x)=\frac{e^{t x} d F_{X}(x)}{\mathbb{E}\left[e^{t X}\right]}, \quad t \in \mathbb{R}
$$

its Esscher transform with parameter $t$. Furthermore, we define the real-valued function $\psi_{X}(\cdot)$ as follows:

$$
\psi_{X}(t)=\int_{(-\infty,+\infty)} x d F_{X}^{(t)}(x)=\frac{\mathbb{E}\left[X e^{t X}\right]}{\mathbb{E}\left[e^{t X}\right]} .
$$

The number $\psi_{X}(t)$ is known as the Esscher premium with parameter $t$, see Bühlmann (1980) and Goovaerts, De Vijlder \& Haezendonck (1984). Since by (1),

$$
\frac{d}{d t}\left(t \varphi_{X}(t)\right)=\psi_{X}(t)
$$

when $t \neq 0$, it follows that

$$
\varphi_{X}(t)=\frac{1}{t} \int_{0}^{t} \psi_{X}(s) d s, \quad t \neq 0
$$

As is well-known, both the Esscher premiums and the exponential premiums increase with their parameter. We remark for later reference that

$$
\lim _{t \rightarrow-\infty} \varphi_{X}(t)=\min [X]=\lim _{t \rightarrow-\infty} \psi_{X}(t)
$$

and that

$$
\lim _{t \rightarrow+\infty} \varphi_{X}(t)=\max [X]=\lim _{t \rightarrow+\infty} \psi_{X}(t) .
$$

For notational convenience, we write in the sequel $\varphi_{X}(t)$ and $\psi_{X}(t)$ also when $t=-\infty$ or $t=+\infty$, understanding the limits for $t \rightarrow-\infty$ or $t \rightarrow+\infty$ in that case. We introduce the notions of exponential order and Laplace transform order. We say that a r.v. $X$ is smaller than a r.v. $Y$ in exponential order if

$$
\mathbb{E}\left[e^{t X}\right] \leq \mathbb{E}\left[e^{t Y}\right], \quad t \geq 0
$$

Furthermore, we say that a r.v. $X$ is smaller than a r.v. $Y$ in Laplace transform order if

$$
\mathbb{E}\left[e^{t X}\right] \geq \mathbb{E}\left[e^{t Y}\right], \quad t \leq 0 .
$$

We write $X \leq_{\mathrm{e}} Y$ and $X \leq_{\mathrm{Lt}} Y$, respectively. Note that $X \leq_{\mathrm{e}} Y$ is equivalent to $-Y \leq_{\mathrm{Lt}}$ $-X$. In the sequel, we look at pairs of r.v.'s $X$ and $Y$ such that both $X \leq_{\mathrm{e}} Y$ and $X \leq_{\mathrm{Lt}} Y$ 
(or equivalently $X \leq_{\mathrm{e}} Y$ and $-Y \leq_{\mathrm{e}}-X$ ). Clearly, the twofold condition $X \leq_{\mathrm{e}} Y$ and $X \leq_{\mathrm{Lt}} Y$ is also equivalent to the condition $\varphi_{X}(t) \leq \varphi_{Y}(t)$ for all $t$. The interested reader is referred to Denuit (2001) for a further treatment of the two notions of stochastic order.

We denote by the functional $\pi[\cdot]$ a risk measure that assigns a real number to a given r.v. Then we introduce the set $\mathbb{S}$ of axioms that $\pi[\cdot]$ must satisfy:

A1. If $\varphi_{X}(t) \leq \varphi_{Y}(t)$ for all $t$ then $\pi[X] \leq \pi[Y]$

A2. $\pi[c]=c$, for all real $c$;

A3. $\pi[X+Y]=\pi[X]+\pi[Y]$ when $X$ and $Y$ are independent;

A4. If $X_{n}$ converges weakly to $X$, with $\min \left[X_{n}\right] \rightarrow \min [X]$ and $\max \left[X_{n}\right] \rightarrow \max [X]$, then $\lim _{n \rightarrow+\infty} \pi\left[X_{n}\right]=\pi[X]$.

Clearly, $X$ and $Y$ have uniformly ordered exponential premiums (also for risk-loving exponential decision makers), or what is the same, moment generating functions (mgf's) crossing at 0 , if $X$ is stochastically dominated by $Y$, written as $X \leq_{\text {st }} Y$. Therefore, axiom A1 guarantees monotonicity of the risk measure $\pi[\cdot]$. Though stochastic order cannot hold for different distributions that have the same expectation, pairs with ordered exponential premiums and yet the same expectation do exist. Consider for instance the r.v.'s $X$ and $Y$ with $\mathbb{P}[X=1]=\frac{2}{3}=1-\mathbb{P}[X=-2]$ and $Y=-X$. It follows from Taylor expansions for their mgf's that if $X$ and $Y$ have ordered exponential premiums and equal expectations, they must have the same variance as well. Moreover, if $X$ and $Y$ have the first three moments in common, the fourth moment ("peakedness") must also be equal if $X$ and $Y$ have ordered exponential premiums. Notice that $X$ and $Y$ having ordered exponential premiums implies that $X \leq_{\mathrm{cx}} Y$ cannot hold; here as usual we write $X \leq_{\mathrm{cx}} Y$ if for any convex function $f(\cdot)$ it holds that $\mathbb{E}[f(X)] \leq \mathbb{E}[f(Y)]$.

Note that $c$ plays two roles in axiom A2: a r.v. degenerated at $c$ on the left-hand side and a real number on the right-hand side. In the economic literature, axiom A2 is known as the certainty equivalence condition. One can regard axiom A3, which imposes additivity for independent random variables, as the most "characteristic" axiom. Additivity of the risk measure for independent r.v.'s is particularly desirable in the context of premium calculation and allocation from top down for a portfolio consisting of independent policies; see Bühlmann (1985) or Kaas et al. (2001), section 5.2. Axiom A4 can be regarded as a continuity condition on the risk measure $\pi[\cdot]$.

To characterize the mixed Esscher principle, Gerber \& Goovaerts (1981) impose the same axioms A2 and A3, and a weaker version of axiom A1. Axiom A4 is imposed, too, 
though not stated explicitly. Their weaker version of axiom A1 says that if $\psi_{X}(t) \leq \psi_{Y}(t)$ for all $t$, then $\pi[X] \leq \pi[Y]$. From (4) it follows that if $\psi_{X}(t) \leq \psi_{Y}(t)$ for all $t$, then also $\varphi_{X}(t) \leq \varphi_{Y}(t)$ for all $t$. Note that the converse is not true. Note furthermore that while $X \leq_{\text {st }} Y$ implies ordered exponential premiums, it does not necessarily imply ordered Esscher premiums. Therefore, the mixed Esscher principle is not monotonic in general.

Below we will restate the four axioms using the one-to-one correspondence between the cdf of $X$ and the function $\varphi_{X}(\cdot)$. For that purpose, we first introduce several concepts. In the following, we arbitrarily fix a defective, continuous r.v. $T_{0}$ with a strictly increasing cdf $F_{T_{0}}(\cdot)$, supported on $[-\infty,+\infty]$ and having positive jumps at both $-\infty$ and $+\infty$. We consider $\varphi_{X}\left(T_{0}\right)$, where the function $\varphi_{X}(\cdot)$ is as defined in $(1)$. Clearly, because $\varphi_{X}(\cdot)$ depends on the cdf of $X$ rather than on the particular r.v. $X$, we can assume throughout without loss of generality that $T_{0}$ is independent of all indices used. Hence, $\varphi_{X}\left(T_{0}\right)$ can be expressed as

$$
\varphi_{X}\left(T_{0}\right)=\frac{1}{T_{0}} \log \mathbb{E}_{X}\left[e^{T_{0} X}\right]
$$

The r.v. $\varphi_{X}\left(T_{0}\right)$ can be regarded as an exponential premium with random parameter $T_{0}$. We remark that there exists a one-to-one correspondence between $X$ and $\varphi_{X}\left(T_{0}\right)$ in the sense that two r.v.'s $X$ and $Y$ are equal in distribution if and only if $\varphi_{X}\left(T_{0}\right)=\varphi_{Y}\left(T_{0}\right)$, almost surely (a.s.).

Next, we introduce comonotonicity of a random vector.

Definition 1 A random vector $\left(X_{1}, \ldots, X_{n}\right)$ is comonotonic if there exists a r.v. $T$ and non-decreasing functions $f_{i}, i=1, \ldots, n$, such that

$$
\left(X_{1}, \ldots, X_{n}\right)=\left(f_{1}(T), \ldots, f_{n}(T)\right), \quad \text { in distribution }
$$

We refer to Dhaene et al. (2002a, 2002b) for an extensive treatment of comonotonicity and its applications in actuarial science.

We introduce the class $\Phi_{T_{0}}$ defined by

$$
\Phi_{T_{0}}=\left\{\varphi_{X}\left(T_{0}\right) \mid X \text { a bounded r.v. }\right\}
$$

The class $\Phi_{T_{0}}$ contains all r.v.'s $\varphi_{X}\left(T_{0}\right)$ generated by bounded r.v.'s $X$. Then, we define for the risk measure $\pi[\cdot]$ satisfying the set $\mathbb{S}$ of axioms, the functional $\rho_{T_{0}}: \Phi_{T_{0}} \rightarrow \mathbb{R}$ that assigns the real number $\pi[X]$ to the r.v. $\varphi_{X}\left(T_{0}\right)$, i.e.,

$$
\rho_{T_{0}}\left[\varphi_{X}\left(T_{0}\right)\right]=\pi[X]
$$


If (and only if) $\pi[\cdot]$ satisfies the set $\mathbb{S}$ of axioms, the functional $\rho_{T_{0}}[\cdot]$ satisfies the following set $\mathbb{S}^{\prime}$ of axioms:

A1'. If $\varphi_{X}\left(T_{0}\right) \leq \varphi_{Y}\left(T_{0}\right)$ a.s., then $\rho_{T_{0}}\left[\varphi_{X}\left(T_{0}\right)\right] \leq \rho_{T_{0}}\left[\varphi_{Y}\left(T_{0}\right)\right]$

A2'. $\rho_{T_{0}}\left[\varphi_{c}\left(T_{0}\right)\right]=c$, for all real $c$;

A3'. $\rho_{T_{0}}\left[\varphi_{X}\left(T_{0}\right)+\varphi_{Y}\left(T_{0}\right)\right]=\rho_{T_{0}}\left[\varphi_{X}\left(T_{0}\right)\right]+\rho_{T_{0}}\left[\varphi_{Y}\left(T_{0}\right)\right]$

A4'. If $\varphi_{X_{n}}\left(T_{0}\right)$ converges a.s. to $\varphi_{X}\left(T_{0}\right)$, then $\lim _{n \rightarrow+\infty} \rho_{T_{0}}\left[\varphi_{X_{n}}\left(T_{0}\right)\right]=\rho_{T_{0}}\left[\varphi_{X}\left(T_{0}\right)\right]$.

To verify that A4' is equivalent to A4 we state the following lemma:

Lemma 2 For a given sequence $\left\{X_{n}\right\}$ of bounded r.v.'s and a bounded (limit) r.v. $X$, it holds that $X_{n}$ converges weakly to $X$, with $\min \left[X_{n}\right] \rightarrow \min [X]$ and $\max \left[X_{n}\right] \rightarrow \max [X]$, if and only if $\varphi_{X_{n}}\left(T_{0}\right)$ converges a.s. to $\varphi_{X}\left(T_{0}\right)$.

Proof of "only if" part: Since $X_{n}$ converges weakly to $X$, with $\min \left[X_{n}\right] \rightarrow \min [X]$ and $\max \left[X_{n}\right] \rightarrow \max [X]$, it is not difficult to see that there exists some constant $c>0$ such that $\left|X_{n}\right| \leq c$ and $|X| \leq c$ hold a.s. Hence, by the dominated convergence theorem, the relation

$$
\lim _{n \rightarrow+\infty} \varphi_{X_{n}}(t)=\varphi_{X}(t)
$$

holds for all $t \in[-\infty,+\infty]$. This proves that $\varphi_{X_{n}}\left(T_{0}\right)$ converges a.s. to $\varphi_{X}\left(T_{0}\right)$.

Proof of "if" part: Since $\varphi_{X_{n}}\left(T_{0}\right)$ converges a.s. to $\varphi_{X}\left(T_{0}\right)$ and the events $\left\{T_{0}=-\infty\right\}$ and $\left\{T_{0}=+\infty\right\}$ have positive probabilities, the convergences $\min \left[X_{n}\right] \rightarrow \min [X]$ and $\max \left[X_{n}\right] \rightarrow \max [X]$ follow immediately from (5) and (6). By the continuity of mgftransformations (see e.g., Theorem 2, Chapter XIII, p. 431 of Feller (1971)), to prove that $X_{n}$ converges weakly to $X$, it suffices to prove that relation (13) holds for all real $t$. For this purpose we recall the assumptions on the random variable $T_{0}$ and the monotonicity of the function $\varphi_{X_{n}}(\cdot)$. By the a.s. convergence of $\varphi_{X_{n}}\left(T_{0}\right)$ to $\varphi_{X}\left(T_{0}\right)$, it holds for any $\varepsilon>0$ that

$$
\lim _{n \rightarrow+\infty} \mathbb{E}\left[\varphi_{X_{n}}\left(T_{0}\right) 1_{\left\{t<T_{0} \leq t+\varepsilon\right\}}\right]=\mathbb{E}\left[\varphi_{X}\left(T_{0}\right) 1_{\left\{t<T_{0} \leq t+\varepsilon\right\}}\right],
$$

where as usual, we denote by $1_{A}$ the indicator function of event $A$. Hence,

$$
\begin{aligned}
\varphi_{X_{n}}(t) & \leq \frac{1}{\mathbb{P}\left[t<T_{0} \leq t+\varepsilon\right]} \int_{t}^{t+\varepsilon} \varphi_{X_{n}}(u) d F_{T_{0}}(u) \\
& \rightarrow \frac{1}{\mathbb{P}\left[t<T_{0} \leq t+\varepsilon\right]} \int_{t}^{t+\varepsilon} \varphi_{X}(u) d F_{T_{0}}(u) \\
& \leq \varphi_{X}(t+\varepsilon) .
\end{aligned}
$$


Then, by the arbitrariness of $\varepsilon>0$ and the continuity of the function $\varphi_{X}(\cdot)$, it follows that

$$
\limsup _{n \rightarrow+\infty} \varphi_{X_{n}}(t) \leq \varphi_{X}(t)
$$

Similarly, we can prove that

$$
\liminf _{n \rightarrow+\infty} \varphi_{X_{n}}(t) \geq \varphi_{X}(t) .
$$

This proves that (13) holds for all real $t$, which ends the proof of Lemma 2.

Note that axiom A3' is the comonotonic image of axiom A3, recalling that $\varphi_{X}(\cdot)$ is a non-decreasing function. Indeed, the additivity of the risk measure $\pi[\cdot]$ for independent r.v.'s $X$ and $Y$ in axiom A3 corresponds to the additivity of the functional $\rho_{T_{0}}[\cdot]$ for the comonotonic r.v.'s $\varphi_{X}\left(T_{0}\right)$ and $\varphi_{Y}\left(T_{0}\right)$ in axiom A3'.

Let us consider the functional $\rho_{T_{0}}[\cdot]$ in further detail. We define $p_{1}=F_{T_{0}}(-\infty)$ and $1-p_{2}=F_{T_{0}}(+\infty)$. Let $A_{1}$ be the event $\left\{T_{0}=-\infty\right\}, A_{2}$ be the event $\left\{-\infty<T_{0}<+\infty\right\}$ and $A_{3}$ be the event $\left\{T_{0}=+\infty\right\}$. We define the r.v. $U\left(T_{0}\right)$ as follows:

$$
U\left(T_{0}\right)=1_{A_{1}} U_{1}+1_{A_{2}} F_{T_{0}}\left(T_{0}\right)+1_{A_{3}} U_{3},
$$

where $U_{1}$ is uniformly distributed on $\left(0, p_{1}\right)$ and independent of $1_{A_{1}}$, and $U_{3}$ is uniformly distributed on $\left(1-p_{2}, 1\right)$ and independent of $1_{A_{3}}$. Then $U\left(T_{0}\right)$ is uniformly distributed on $(0,1)$. Notice that $U(s)<U(t)$ a.s. whenever $s<t$, including the cases when $s=-\infty$ or $t=+\infty$. It is well-known that for a given r.v. $V$ it holds that

$$
V=F_{V}^{-1}\left(U\left(T_{0}\right)\right), \quad \text { in distribution, }
$$

where as usual we denote by $F_{V}^{-1}(\cdot)$ the generalized inverse cdf of $V$, defined by

$$
F_{V}^{-1}(p)=\inf \left\{x \in \mathbb{R} \mid F_{V}(x) \geq p\right\} .
$$

We remark for later reference that a r.v. $V$ is stochastically dominated by a r.v. $W$ if and only if $F_{V}^{-1}\left(U\left(T_{0}\right)\right) \leq F_{W}^{-1}\left(U\left(T_{0}\right)\right)$, a.s.

Note that for any given bounded r.v. $X$ there exists a r.v. $V$ with a strictly increasing cdf and the same support as $X$ such that

$$
\varphi_{X}\left(T_{0}\right)=F_{V}^{-1}\left(U\left(T_{0}\right)\right), \quad \text { a.s. }
$$

Notice that, conversely, for a particular bounded r.v. $V$ there may not exist a bounded r.v. $X$ such that

$$
F_{V}^{-1}\left(U\left(T_{0}\right)\right)=\varphi_{X}\left(T_{0}\right), \quad \text { a.s. }
$$


One may verify the latter statement by considering for $V$ a Bernoulli r.v. Consequently, the functional $\rho_{T_{0}}[\cdot]$ defined in (12) is not defined for arbitrary r.v.'s $F_{V}^{-1}\left(U\left(T_{0}\right)\right)$ with $V$ a bounded r.v. To extend the domain of the functional $\rho_{T_{0}}[\cdot]$ to arbitrary bounded r.v.'s $F_{V}^{-1}\left(U\left(T_{0}\right)\right)$, we introduce the class $\Theta_{T_{0}}=\left\{F_{V}^{-1}\left(U\left(T_{0}\right)\right) \mid V\right.$ a bounded r.v. $\}$ of which $\Phi_{T_{0}}$ is a subclass and we impose that $\rho_{T_{0}}[\cdot]: \Theta_{T_{0}} \rightarrow \mathbb{R}$ satisfies the set $\mathbb{S}$ " of axioms, which is the analog of $\mathbb{S}^{\prime}$, given by

A1". (Monotonicity) If $F_{V}^{-1}\left(U\left(T_{0}\right)\right) \leq F_{W}^{-1}\left(U\left(T_{0}\right)\right)$ a.s., then $\rho_{T_{0}}\left[F_{V}^{-1}\left(U\left(T_{0}\right)\right)\right] \leq$ $\rho_{T_{0}}\left[F_{W}^{-1}\left(U\left(T_{0}\right)\right)\right]$

A2". (Certainty Equivalence) $\rho_{T_{0}}[c]=c$, for all real $c$;

A3". (Comonotonic Additivity) $\rho_{T_{0}}\left[F_{V}^{-1}\left(U\left(T_{0}\right)\right)+F_{W}^{-1}\left(U\left(T_{0}\right)\right)\right]=\rho_{T_{0}}\left[F_{V}^{-1}\left(U\left(T_{0}\right)\right)\right]+$ $\rho_{T_{0}}\left[F_{W}^{-1}\left(U\left(T_{0}\right)\right)\right]$;

A4". (Continuity) If $F_{V_{n}}^{-1}\left(U\left(T_{0}\right)\right)$ converges a.s. to $F_{V}^{-1}\left(U\left(T_{0}\right)\right)$, then $\lim _{n \rightarrow+\infty} \rho_{T_{0}}\left[F_{V_{n}}^{-1}\left(U\left(T_{0}\right)\right)\right]=\rho_{T_{0}}\left[F_{V}^{-1}\left(U\left(T_{0}\right)\right)\right]$.

Notice that A1" can be restated as: if $V \leq_{\text {st }} W$, then $\rho_{T_{0}}\left[F_{V}^{-1}\left(U\left(T_{0}\right)\right)\right] \leq \rho_{T_{0}}\left[F_{W}^{-1}\left(U\left(T_{0}\right)\right)\right]$. Furthermore, notice that A4" is equivalent to the condition that if $V_{n}$ converges weakly to $V$, then $\lim _{n \rightarrow+\infty} \rho_{T_{0}}\left[F_{V_{n}}^{-1}\left(U\left(T_{0}\right)\right)\right]=\rho_{T_{0}}\left[F_{V}^{-1}\left(U\left(T_{0}\right)\right)\right]$. However, we prefer to present axioms A1" and A4" in the way we have done above, to demonstrate explicitly that $\mathbb{S}$ " is the analog of $\mathbb{S}^{\prime}$.

Notice that the set $\mathbb{S}$ " of axioms is more restrictive than the set $\mathbb{S}^{\prime}$ of axioms (and hence also more restrictive than the original set $\mathbb{S}$ of axioms) since it imposes conditions on a richer class of r.v.'s. Therefore, formally, the representation theorem presented below establishes an axiomatic characterization of the functional $\rho_{T_{0}}[\cdot]$ instead of for the risk measure $\pi[\cdot]$. On the subclass $\Phi_{T_{0}}, \rho_{T_{0}}\left[\varphi_{X}\left(T_{0}\right)\right]$ coincides with $\pi[X]$ by definition, which will allow us to derive the functional form of $\pi[\cdot]$. To characterize the mixed Esscher principle, Gerber \& Goovaerts (1981) tacitly perform a similar extension to the class of r.v.'s to which the axioms apply.

A representation theorem for the functional $\rho_{T_{0}}[\cdot]$ can be established by using the representation theorem for comonotonic additive risk measures as presented in $\mathrm{Wu} \&$ Wang (2003); see for the original work Greco (1982) (or translated into English: Denneberg (1994)), Schmeidler (1989) and Yaari (1987). Although Wu \& Wang (2003) consider only non-negative r.v.'s, their result also applies to our case where all r.v.'s are real-valued and bounded. To verify this statement note that because of the comonotonic additivity of the 
functional $\rho_{T_{0}}[\cdot]$, it holds that

$$
\begin{aligned}
\rho_{T_{0}}\left[F_{V}^{-1}\left(U\left(T_{0}\right)\right)\right] & =\rho_{T_{0}}\left[F_{V}^{-1}\left(U\left(T_{0}\right)\right)-\min [V]+\min [V]\right] \\
& =\rho_{T_{0}}\left[F_{V}^{-1}\left(U\left(T_{0}\right)\right)-\min [V]\right]+\min [V] .
\end{aligned}
$$

In particular, applying Theorem 3.2 from Wu \& Wang (2003), we derive that under the set $\mathbb{S}$ " of axioms, the functional $\rho_{T_{0}}[\cdot]$ can be represented by

$$
\rho_{T_{0}}\left[F_{V}^{-1}\left(U\left(T_{0}\right)\right)\right]=\int_{(-\infty,+\infty)} v d\left(1-w\left(1-F_{F_{V}^{-1}\left(U\left(T_{0}\right)\right)}(v)\right)\right)
$$

in which the function $w(\cdot):[0,1] \rightarrow[0,1]$ is non-decreasing, right continuous and satisfies $w(0)=0$ and $w(1)=1$. Then we state the following theorem:

Theorem 3 The functional $\rho_{T_{0}}[\cdot]$ satisfies the set $\mathbb{S}$ " of axioms if and only if there exists some non-decreasing function $G:[-\infty,+\infty] \rightarrow[0,1]$ such that

$\rho_{T_{0}}\left[F_{V}^{-1}\left(U\left(T_{0}\right)\right)\right]=G(-\infty) F_{V}^{-1}\left(p_{1}\right)+\int_{(-\infty,+\infty)} F_{V}^{-1}(U(t)) d G(t)+(1-G(+\infty)) F_{V}^{-1}\left(1-p_{2}\right)$.

On the subclass $\Phi_{T_{0}}$, the functional $\rho_{T_{0}}[\cdot]$ (and consequently the risk measure $\pi[\cdot]$ ) can be represented by a mixture of exponential premiums, i.e.,

$$
\begin{aligned}
\pi[X] & =\int_{[-\infty,+\infty]} \varphi_{X}(t) d G(t) \\
& =G(-\infty) \min [X]+\int_{(-\infty,+\infty)} \varphi_{X}(t) d G(t)+(1-G(+\infty)) \max [X] .
\end{aligned}
$$

Proof: Consider representation (17). Since $F_{F_{V}^{-1}\left(U\left(T_{0}\right)\right)}(v)=F_{V}(v)$ and the generalized inverse cdf $F_{V}^{-1}(\cdot)$ is appropriately defined, substituting $v=F_{V}^{-1}\left(F_{T_{0}}(t)\right)$ gives

$$
\rho_{T_{0}}\left[F_{V}^{-1}\left(U\left(T_{0}\right)\right)\right]=\int_{[-\infty,+\infty]} F_{V}^{-1}\left(F_{T_{0}}(t)\right) d\left(1-w\left(1-F_{T_{0}}(t)\right)\right) .
$$

Hence, by the definition of $U\left(T_{0}\right)$, we have that

$$
\begin{aligned}
\rho_{T_{0}}\left[F_{V}^{-1}\left(U\left(T_{0}\right)\right)\right]= & \left(1-w\left(1-p_{1}\right)\right) F_{V}^{-1}\left(p_{1}\right) \\
& +\int_{(-\infty,+\infty)} F_{V}^{-1}(U(t)) d\left(1-w\left(1-F_{T_{0}}(t)\right)\right) \\
& +w\left(p_{2}\right) F_{V}^{-1}\left(1-p_{2}\right) .
\end{aligned}
$$

Then, we define the function $G:[-\infty,+\infty] \rightarrow[0,1]$ as follows:

$$
G(t)=1-w\left(1-F_{T_{0}}(t)\right)
$$


Since $w(\cdot)$ is non-decreasing, we find that $G(\cdot)$ is non-decreasing as well. Notice that if $V$ corresponds to a given bounded r.v. $X$ in the sense of $(15)$, then $F_{V}^{-1}\left(p_{1}\right)=\min [X]$ and $F_{V}^{-1}\left(1-p_{2}\right)=\max [X]$. Hence, on the subclass $\Phi_{T_{0}}$, we have that

$$
\rho_{T_{0}}\left[\varphi_{X}\left(T_{0}\right)\right]=\int_{[-\infty,+\infty]} \varphi_{X}(t) d G(t) .
$$

Now representation (18) follows from equality (12).

One may regard the mixture function $G(\cdot)$ as a cdf, possibly defective with "mass" at both endpoints of its domain. Consequently, the risk measure $\pi[\cdot]$ derived in Theorem 3 can be regarded as the expectation of an exponential premium with random parameter. Here the expectation is not calculated with respect to the real probability distribution of the random parameter but with respect to a transformed probability distribution, see (19). Note the similarity to derivative pricing in arbitrage free financial markets, where derivative prices can be expressed as expectations calculated with respect to an equivalent martingale measure rather than with respect to the real probability measure. We state the following corollary:

Corollary 4 The risk measure $\pi[\cdot]$ in (18) can be represented by a unimodal mixture of Esscher premiums, i.e., there exists some non-decreasing function $H:[-\infty,+\infty] \rightarrow[0,1]$, concave on $(0,+\infty)$ and convex on $(-\infty, 0)$ such that

$$
\begin{aligned}
\pi[X] & =\int_{[-\infty,+\infty]} \psi_{X}(t) d H(t) \\
& =H(-\infty) \min [X]+\int_{(-\infty,+\infty)} \frac{\mathbb{E}\left[X e^{t X}\right]}{\mathbb{E}\left[e^{t X}\right]} d H(t)+(1-H(+\infty)) \max [X] .
\end{aligned}
$$

Proof: We will show that representation (20) is equivalent to representation (18). Consider representation (18). We define a function $M(\cdot)$ as follows:

$$
d M(t)=\frac{1}{t} d G(t), t \neq 0, \quad M(-\infty)=M(+\infty)=0 .
$$

Notice that $M(\cdot)$ is non-decreasing on $(0,+\infty)$ and non-increasing on $(-\infty, 0)$, while $M(0)$ is irrelevant. By substitution of (21) in (18) we obtain

$$
\begin{aligned}
\pi[X]= & G(-\infty) \min [X]+\int_{(-\infty, 0) \cup(0,+\infty)} \log \mathbb{E}\left[e^{t X}\right] d M(t)+(1-G(+\infty)) \max [X] \\
& +\mathbb{E}[X](G(0+)-G(0-))
\end{aligned}
$$


Integration by parts of the above representation yields

$$
\begin{aligned}
\pi[X]= & G(-\infty) \min [X]-\int_{(-\infty, 0) \cup(0,+\infty)} \frac{\mathbb{E}\left[X e^{t X}\right]}{\mathbb{E}\left[e^{t X}\right]} M(t) d t+(1-G(+\infty)) \max [X] \\
& +\mathbb{E}[X](G(0+)-G(0-)),
\end{aligned}
$$

where it is not difficult to verify that the boundary terms obtained by performing the integration by parts of the integral in (22) vanish. Then we define a function $H(\cdot)$ as follows:

$$
d H(t)=-M(t) d t, t \neq 0, \quad H(-\infty)=G(-\infty) ; \quad H(+\infty)=G(+\infty) .
$$

Notice that because $-M(\cdot)$ is non-increasing on $(0,+\infty)$ and non-decreasing on $(-\infty, 0)$, $H(\cdot)$ is concave on $(0,+\infty)$ and convex on $(-\infty, 0)$. Then, by substitution of $(24)$ in $(23)$ we obtain

$$
\begin{aligned}
\pi[X]= & H(-\infty) \min [X]+\int_{(-\infty, 0) \cup(0,+\infty)} \frac{\mathbb{E}\left[X e^{t X}\right]}{\mathbb{E}\left[e^{t X}\right]} d H(t)+(1-H(+\infty)) \max [X] \\
& +\mathbb{E}[X](G(0+)-G(0-)) .
\end{aligned}
$$

Now it suffices to verify that $G(0+)-G(0-)$ is equal to $H(0+)-H(0-)$. It is not difficult to see by substitution of $(21)$ in $(24)$ that

$$
H(0+)=\lim _{x \downarrow 0}\left(-\int_{(x,+\infty)} \int_{(t,+\infty)} \frac{1}{s} d G(s) d t\right)+G(+\infty) .
$$

Observing that the double integral is continuous in $x$ and changing the order of integration yields

$$
H(0+)=-\int_{(0,+\infty)} \int_{(0, s)} \frac{1}{s} d t d G(s)+G(+\infty)=G(0+) .
$$

Similarly one can verify that $H(0-)=G(0-)$. This proves the stated result.

The mixture function $H(\cdot)$ can be regarded as a cdf, unimodal at 0 and possibly defective.

Remark 5 A direct proof of the proposition that on $\Phi_{T_{0}}$ the functional $\rho_{T_{0}}[\cdot]$ (and consequently the risk measure $\pi[\cdot])$ can be represented by a unimodal mixture of Esscher premiums, without borrowing the result of Theorem 3, can be obtained by considering the following decomposition:

$$
\begin{aligned}
\varphi_{X}\left(T_{0}\right)= & \int_{(0,+\infty)} \frac{\left(T_{0}-t\right)_{+}}{T_{0}} 1_{\left\{T_{0}>0\right\}} d \psi_{X}(t) \\
& +\int_{(-\infty, 0)} \frac{\left(t-T_{0}\right)_{+}}{T_{0}} 1_{\left\{T_{0}<0\right\}} d \psi_{X}(t) \\
& +\psi_{X}(0) .
\end{aligned}
$$


Proof: We introduce a sequence of partitions $P_{n}$ given by

$$
P_{n}=\left\{t_{-n, n}, t_{-n+1, n}, \ldots, t_{-1, n}, t_{0, n}, t_{1, n}, \ldots, t_{n-1, n}, t_{n, n}\right\}, \quad n=1,2, \ldots,
$$

in which $t_{m, n}, m=-n, \ldots, n$ are real numbers satisfying $t_{-n, n}<t_{-n+1, n}<\ldots<t_{-1, n}<$ $t_{0, n}=0<t_{1, n}<\ldots<t_{n-1, n}<t_{n, n}$, with $\lim _{n \rightarrow+\infty} \max _{-n+1 \leq m \leq n}\left|t_{m, n}-t_{m-1, n}\right|=0$ and furthermore $t_{-n, n} \rightarrow-\infty$ and $t_{n, n} \rightarrow+\infty$ if $n \rightarrow+\infty$. We let the partitions $P_{n}$ be increasing in the sense that $P_{1} \subset P_{2} \subset \ldots$ Then, (25) can be expressed as follows:

$$
\begin{aligned}
\varphi_{X}\left(T_{0}\right)= & \lim _{n \rightarrow+\infty} \sum_{j=1}^{n}\left(\psi_{X}\left(t_{j, n}\right)-\psi_{X}\left(t_{j-1, n}\right)\right) \frac{\left(T_{0}-t_{j, n}\right)_{+}}{T_{0}} 1_{\left\{T_{0}>0\right\}} \\
& +\lim _{n \rightarrow+\infty} \sum_{j=-n+1}^{-1}\left(\psi_{X}\left(t_{j, n}\right)-\psi_{X}\left(t_{j-1, n}\right)\right) \frac{\left(t_{j-1, n}-T_{0}\right)_{+}}{T_{0}} 1_{\left\{T_{0}<0\right\}} \\
& +\psi_{X}(0) .
\end{aligned}
$$

Applying the functional $\rho_{T_{0}}[\cdot]$ to both sides of the last equation, it follows by A3", A4" and A2", respectively, that

$$
\begin{aligned}
\rho_{T_{0}}\left[\varphi_{X}\left(T_{0}\right)\right]= & \int_{(0,+\infty)} \rho_{T_{0}}\left[\frac{\left(T_{0}-t\right)_{+}}{T_{0}} 1_{\left\{T_{0}>0\right\}}\right] d \psi_{X}(t) \\
& +\int_{(-\infty, 0)} \rho_{T_{0}}\left[\frac{\left(t-T_{0}\right)_{+}}{T_{0}} 1_{\left\{T_{0}<0\right\}}\right] d \psi_{X}(t) \\
& +\psi_{X}(0) .
\end{aligned}
$$

Then the mixture function $H(\cdot)$ can be expressed as follows:

$$
H(t)= \begin{cases}1-\rho_{T_{0}}\left[\frac{\left(T_{0}-t\right)_{+}}{T_{0}} 1_{\left\{T_{0}>0\right\}}\right], & t>0 \\ -\rho_{T_{0}}\left[\frac{\left(t-T_{0}\right)_{+}}{T_{0}} 1_{\left\{T_{0}<0\right\}}\right], & t<0 \\ H(0+), & t=0 .\end{cases}
$$

By substituting (28) into (27), we obtain representation (20) after integration by parts. It is not difficult to verify from $(28)$ that $H(\cdot)$ is non-decreasing. It remains to prove that $H(\cdot)$ is concave on $(0,+\infty)$ and convex on $(-\infty, 0)$. Let $a>0$ and $b<0$. Clearly it holds that

$$
\left(T_{0}+T_{0}-2 t-2 a\right)_{+} \leq\left(T_{0}-t\right)_{+}+\left(T_{0}-t-2 a\right)_{+}, \quad \text { a.s. }
$$

and that

$$
\left(2 t+2 b-T_{0}-T_{0}\right)_{+} \leq\left(t-T_{0}\right)_{+}+\left(t+2 b-T_{0}\right)_{+}, \quad \text { a.s. }
$$


and hence that for all $t>0$

$$
\frac{2\left(T_{0}-t-a\right)_{+}}{T_{0}} 1_{\left\{T_{0}>0\right\}} \leq \frac{\left(T_{0}-t\right)_{+}+\left(T_{0}-t-2 a\right)_{+}}{T_{0}} 1_{\left\{T_{0}>0\right\}}, \quad \text { a.s. }
$$

and that for all $t<0$

$$
\frac{2\left(t+b-T_{0}\right)_{+}}{T_{0}} 1_{\left\{T_{0}<0\right\}} \geq \frac{\left(t-T_{0}\right)_{+}+\left(t+2 b-T_{0}\right)_{+}}{T_{0}} 1_{\left\{T_{0}<0\right\}}, \quad \text { a.s. }
$$

Then, we obtain by application of A1" that

$$
\rho_{T_{0}}\left[\frac{2\left(T_{0}-t-a\right)_{+}}{T_{0}} 1_{\left\{T_{0}>0\right\}}\right] \leq \rho_{T_{0}}\left[\frac{\left(T_{0}-t\right)_{+}+\left(T_{0}-t-2 a\right)_{+}}{T_{0}} 1_{\left\{T_{0}>0\right\}}\right], \quad t>0
$$

and that

$$
\rho_{T_{0}}\left[\frac{2\left(t+b-T_{0}\right)_{+}}{T_{0}} 1_{\left\{T_{0}<0\right\}}\right] \geq \rho_{T_{0}}\left[\frac{\left(t-T_{0}\right)_{+}+\left(t+2 b-T_{0}\right)_{+}}{T_{0}} 1_{\left\{T_{0}<0\right\}}\right], \quad t<0 .
$$

Now recall (28) to verify that

$$
2 H(t+a) \geq H(t)+H(t+2 a), \quad t>0
$$

and that

$$
2 H(t+b) \leq H(t)+H(t+2 b), \quad t<0,
$$

which proves that $H(\cdot)$ is concave on $(0,+\infty)$ and convex on $(-\infty, 0)$.

Although the proof along this line is perhaps less straightforward, it has the nice feature that the mixture function $H(\cdot)$ can be expressed explicitly in terms of the risk measure $\pi[\cdot]$ applied to a special Bernoulli r.v. To see this, we denote by $B_{p, z}$, with $p, z>0$, a Bernoulli r.v. defined by

$$
B_{p, z}= \begin{cases}z, & \text { with } \mathbb{P}\left[B_{p, z}=z\right]=p \\ 0, & \text { with } \mathbb{P}\left[B_{p, z}=0\right]=1-p .\end{cases}
$$

In the following we consider the r.v. $B_{p(t, z), z}$ for the specific choice of

$$
p(t, z)=\frac{q e^{-t z}}{1-q+q e^{-t z}}
$$

for some $q \in(0,1)$ and some $z>0$. Since

$$
\lim _{z \rightarrow+\infty} \frac{1}{z} \varphi_{B_{p(t, z), z}}\left(T_{0}\right)= \begin{cases}\frac{\left(T_{0}-t\right)_{+}}{T_{0}} 1_{\left\{T_{0}>0\right\}}, & \text { a.s. if } t>0 \\ 1+\frac{\left(t-T_{0}\right)_{+}}{T_{0}} 1_{\left\{T_{0}<0\right\}}, & \text { a.s. if } t<0\end{cases}
$$


the function $H(\cdot)$ can be expressed as

$$
H(t)= \begin{cases}1-\rho_{T_{0}}\left[\lim _{z \rightarrow+\infty} \frac{1}{z} \varphi_{B_{p(t, z), z}}\left(T_{0}\right)\right], & t \neq 0 \\ H(0+), & t=0 .\end{cases}
$$

Because of (12) and axiom A4 the function $H(\cdot)$ can also be expressed as

$$
H(t)= \begin{cases}1-\lim _{z \rightarrow+\infty} \frac{1}{z} \pi\left[B_{p(t, z), z}\left(T_{0}\right)\right], & t \neq 0 \\ H(0+), & t=0 .\end{cases}
$$

Hence, we find that the function $H(\cdot)$ can be regarded as the risk perception with respect to a special Bernoulli r.v.

Remark 6 Let (20) be rewritten as

$$
\pi[X]=\int_{[-\infty,+\infty]} \frac{\mathbb{E}\left[X e^{t X}\right]}{\mathbb{E}\left[e^{t X}\right]} d H(t)
$$

This representation allows us to express $\pi[\cdot]$ as $\pi[X]=\mathbb{E}^{*}[X]$, where the expectation is calculated using the differential

$$
d F_{X}^{(H(\cdot))}(x)=\left(\int_{t \in[-\infty,+\infty]} \frac{e^{t x} d H(t)}{\mathbb{E}\left[e^{t X}\right]}\right) d F_{X}(x) .
$$

We state the following two corollaries without proof:

Corollary 7 Suppose that $A 1$ is strengthened to "if $X \leq_{\mathrm{e}} Y$, then $\pi[X] \leq \pi[Y]$," $A_{4}$ is strengthened to "if $X_{n}$ converges weakly to $X$, with $\max \left[X_{n}\right] \rightarrow \max [X]$, then $\lim _{n \rightarrow+\infty} \pi\left[X_{n}\right]=\pi[X]$," while A2 and A3 remain unchanged. Then the functional $\rho_{T_{0}}[\cdot]$ satisfies the corresponding modified set $\mathbb{S}$ " of axioms if and only if there exists some nondecreasing function $G:[0,+\infty] \rightarrow[0,1]$ such that

$$
\rho_{T_{0}}\left[F_{V}^{-1}\left(U\left(T_{0}\right)\right)\right]=G(0) F_{V}^{-1}\left(p_{1}\right)+\int_{(0,+\infty)} F_{V}^{-1}(U(t)) d G(t)+(1-G(+\infty)) F_{V}^{-1}\left(1-p_{2}\right) .
$$

On the subclass $\Phi_{T_{0}}$, the functional $\rho_{T_{0}}[\cdot]$ (and consequently the risk measure $\pi[\cdot]$ ) can be represented by

$$
\begin{aligned}
\pi[X] & =\int_{[0,+\infty]} \varphi_{X}(t) d G(t) \\
& =G(0) \mathbb{E}[X]+\int_{(0,+\infty)} \varphi_{X}(t) d G(t)+(1-G(+\infty)) \max [X] .
\end{aligned}
$$


Corollary 8 Suppose that $A 1$ is strengthened to "if $X \leq_{\mathrm{Lt}} Y$, then $\pi[X] \leq \pi[Y]$," A4 is strengthened to "if $X_{n}$ converges weakly to $X$, with $\min \left[X_{n}\right] \rightarrow \min [X]$, then $\lim _{n \rightarrow+\infty} \pi\left[X_{n}\right]=\pi[X]$," while A2 and A3 remain unchanged. Then the functional $\rho_{T_{0}}[\cdot]$ satisfies the corresponding modified set $\mathbb{S}$ " of axioms if and only if there exists some nondecreasing function $G:[-\infty, 0] \rightarrow[0,1]$ such that

$$
\rho_{T_{0}}\left[F_{V}^{-1}\left(U\left(T_{0}\right)\right)\right]=G(-\infty) F_{V}^{-1}\left(p_{1}\right)+\int_{(-\infty, 0)} F_{V}^{-1}(U(t)) d G(t)+(1-G(0)) F_{V}^{-1}\left(1-p_{2}\right) .
$$

On the subclass $\Phi_{T_{0}}$, the functional $\rho_{T_{0}}[\cdot]$ (and consequently the risk measure $\pi[\cdot]$ ) can be represented as

$$
\begin{aligned}
\pi[X] & =\int_{[-\infty, 0]} \varphi_{X}(t) d G(t) \\
& =G(-\infty) \min [X]+\int_{(-\infty, 0)} \varphi_{X}(t) d G(t)+(1-G(0)) \mathbb{E}[X] .
\end{aligned}
$$

The proofs of Corollary 7 and Corollary 8 are completely similar to the proof of Theorem 3 , the difference being that $F_{T_{0}}(\cdot)$ is now supported on $[0,+\infty]$ and $[-\infty, 0]$ respectively, rather than on $[-\infty,+\infty]$, in addition to being defective, continuous and strictly increasing.

The condition that $X \leq_{\mathrm{e}} Y$ implies $\pi[X] \leq \pi[Y]$, as imposed in Corollary 7 , has a natural interpretation in the classical ruin model. It is easy to prove that if $X \leq_{\mathrm{e}} Y$, where $X$ and $Y$ represent the i.i.d. claim amounts of two homogeneous Poisson processes with equal Poisson parameter, then the upper bound for the probability of ruin is smaller in case of individual claims $X$ than in case of individual claims $Y$, regardless of the initial capital.

For the particular case in which $G(\cdot)$ is non-decreasing and $G(t)=0$ for $t<0$, i.e., the case of Corollary 7 , the derived risk measure is a mixture of exponential premiums with a non-negative safety loading; see in this context Goovaerts et al. (2003). In the remainder of this paper, we consider some properties of the risk measure derived in Corollary 7 , thus restricting to the case in which $G(t)=0$ for $t<0$, which is reasonable from the viewpoint of premium calculation.

We introduce the notion of stop-loss order. We say that a r.v. $X$ is smaller than a r.v. $Y$ in stop-loss order if $X$ has smaller stop-loss premiums than $Y$, or equivalently, if for any non-decreasing and convex function $f(\cdot)$ it holds that

$$
\mathbb{E}[f(X)] \leq \mathbb{E}[f(Y)]
$$


We write $X \leq_{\text {sl }} Y$. It is a well-known result (see e.g., Kaas et al. (2001), section 10.6) that for any given random vector $\left(X_{1}, \ldots, X_{n}\right)$ and an independent r.v. $U$ uniformly distributed on $(0,1)$ it holds that

$$
X_{1}+\ldots+X_{n} \leq_{\mathrm{sl}} F_{X_{1}}^{-1}(U)+\ldots+F_{X_{n}}^{-1}(U) .
$$

Then we state the following two corollaries:

Corollary 9 If $X \leq_{\mathrm{sl}} Y$, then the risk measure $\pi[\cdot]$ derived in Corollary 7 , satisfies $\pi[X] \leq \pi[Y]$.

Proof: Because $e^{t x}$ is non-decreasing and convex for all $t \geq 0$, we have by the definition of stop-loss order that $X \leq_{\mathrm{sl}} Y$ implies $X \leq_{\mathrm{e}} Y$ and therefore $\pi[X] \leq \pi[Y]$.

Corollary 10 The risk measure $\pi[\cdot]$ derived in Corollary 7 is superadditive for sums of comonotonic r.v.'s, i.e., it holds that

$$
\pi\left[X_{1}\right]+\ldots+\pi\left[X_{n}\right] \leq \pi\left[F_{X_{1}}^{-1}(U)+\ldots+F_{X_{n}}^{-1}(U)\right] .
$$

Proof: Recall (37) and notice that because of the arbitrariness of the random vector $\left(X_{1}, \ldots, X_{n}\right)$, this inequality also applies to the case in which $X_{1}, \ldots, X_{n}$ are independent. Then the proof of the corollary follows by application of Corollary 9 and the additivity property of $\pi[\cdot]$.

\section{Conclusion}

This paper gives an axiomatic characterization of the mixed exponential principle. This premium principle is additive for independent random variables. In contrast to the wellknown mixed Esscher principle, this premium principle is monotonic in the sense that it preserves stochastic dominance. In order to prove the representation theorem, we provide a comonotonic image of the axiom of additivity for independent random variables.

In particular, the following sets of axioms are considered:

A1. If $\varphi_{X}(t) \leq \varphi_{Y}(t)$ for all $t$ then $\pi[X] \leq \pi[Y]$

A2. $\pi[c]=c$, for all real $c$; 
A3. $\pi[X+Y]=\pi[X]+\pi[Y]$ when $X$ and $Y$ are independent;

A4. If $X_{n}$ converges weakly to $X$, with $\min \left[X_{n}\right] \rightarrow \min [X]$ and $\max \left[X_{n}\right] \rightarrow \max [X]$, then $\lim _{n \rightarrow+\infty} \pi\left[X_{n}\right]=\pi[X]$;

and

B1. If $X \leq_{\text {st }} Y$ then $\rho[X] \leq \rho[Y]$;

B2. $\rho[c]=c$, for all real $c$;

B3. $\rho[X+Y]=\rho[X]+\rho[Y]$ when $X$ and $Y$ are comonotonic;

B4. If $X_{n}$ converges weakly to $X$ then $\lim _{n \rightarrow+\infty} \rho\left[X_{n}\right]=\rho[X]$.

It is proved in this paper that the first set of axioms gives rise to a risk measure $\pi[\cdot]$ that can be represented by a mixture of exponential premiums, i.e.,

$$
\pi[X]=G(-\infty) \min [X]+\int_{(-\infty,+\infty)} \varphi_{X}(t) d G(t)+(1-G(+\infty)) \max [X]
$$

for some non-decreasing function $G:[-\infty,+\infty] \rightarrow[0,1]$. It is well-known that the second set of axioms gives rise to a risk measure $\rho[\cdot]$ given by

$$
\rho[X]=\int_{(-\infty,+\infty)} x d\left(1-w\left(1-F_{X}(x)\right)\right),
$$

for some non-decreasing function $w:(-\infty,+\infty) \rightarrow[0,1]$, satisfying $w(0)=0$ and $w(1)=$ 1.

We consider a fixed defective continuous r.v. $T_{0}$ with a strictly increasing $\operatorname{cdf} F_{T_{0}}(\cdot)$, supported on $[-\infty,+\infty]$ and having positive jumps of size $p_{1}$ and $p_{2}$ at $-\infty$ and $+\infty$, respectively. We construct a uniformly distributed r.v. $U\left(T_{0}\right)$ satisfying $U(s)<U(t)$ a.s. whenever $s<t$. Then, we have that $F_{X}^{-1}\left(U\left(T_{0}\right)\right)=X$ in distribution. Furthermore, notice that $\rho[\cdot]$ can then be expressed as

$\rho[X]=\left(1-w\left(1-p_{1}\right)\right) F_{X}^{-1}\left(p_{1}\right)+\int_{(-\infty,+\infty)} F_{X}^{-1}(U(t)) d\left(1-w\left(1-F_{T_{0}}(t)\right)\right)+w\left(p_{2}\right) F_{X}^{-1}\left(1-p_{2}\right)$.

Hence, by the arbitrariness of the function $G(\cdot)$, we have that

$$
\rho[X]=G(-\infty) F_{X}^{-1}\left(p_{1}\right)+\int_{(-\infty,+\infty)} F_{X}^{-1}(U(t)) d G(t)+(1-G(+\infty)) F_{X}^{-1}\left(1-p_{2}\right) .
$$

By comparing (39) and (40), the close connection between $\pi[\cdot]$ and $\rho[\cdot]$ becomes readily apparent. 
Note that if the risk measure (39), which is a weighted average of premiums quoted by exponential decision makers, is to be used as an insurance premium, including in it the premiums asked by risk-lovers is to be regarded as unsound business practice. For this case, (34) is better suited, but it is quite conceivable that economic scenarios can be found where use of (39) is appropriate.

Acknowledgements We are grateful to an anonymous referee for helpful remarks. 


\section{References}

[1] Bühlmann, H. (1980). "An economic premium principle," Astin Bulletin 11, 5260.

[2] Bühlmann, H. (1985). "Premium calculation from top down," Astin Bulletin 15, 89-101.

[3] Denneberg, D. (1994). Non-additive Measure and Integral, Boston: Kluwer Academic Publishers.

[4] Denuit, M. (2001). "Laplace transform ordering of actuarial quantities," Insurance: Mathematics and Economics 29, 83-102.

[5] Dhaene, J., M. Denuit, M.J. Goovaerts, R. Kaas \& D. Vyncke (2002a). "The concept of comonotonicity in actuarial science and finance: Theory," Insurance: Mathematics and Economics 31, 3-33.

[6] Dhaene, J., M. Denuit, M.J. Goovaerts, R. Kaas \& D. Vyncke (2002b). "The concept of comonotonicity in actuarial science and finance: Applications," Insurance: Mathematics and Economics 31, 133-161.

[7] Feller, W. (1971). An Introduction to Probability Theory and Its Applications 2, 2nd edn., New York: Wiley.

[8] Gerber, H.U. (1974). "On additive premium calculation principles," Astin Bulletin $7,215-222$.

[9] Gerber, H.U. (1980). "Credibility for Esscher premiums," Mitteilungen der Vereinigung Schweizerischer Versicherungsmathematiker 3, 307-312.

[10] Gerber, H.U. \& M.J. Goovaerts (1981). "On the representation of additive principles of premium calculation," Scandinavian Actuarial Journal 4, 221-227.

[11] Gerber, H.U. (1981). "The Esscher premium principle: A criticism, Comment," Astin Bulletin 12, 139-140.

[12] Goovaerts, M.J. \& F. De Vijlder (1980). "A note on additive premium calculation principles," Bulletin de l'Association Royal des Actuaires Belges, 74, 89-93.

[13] Goovaerts, M.J., F. De Vijlder \& J. Haezendonck (1984). Insurance Premiums, Amsterdam: North Holland Publishing. 
[14] Goovaerts, M.J., R. Kaas, J. Dhaene \& Q. Tang (2003). "A unified approach to generate risk measures," Astin Bulletin 33, 173-191.

[15] Greco, G. (1982). "Sulla Rappresentazione di Funzionali Mediante Integrali," Rend. Sem. Mat. Univ. Padova 66, 21-42.

[16] Kaas, R., M.J. Goovaerts, J. Dhaene \& M. Denuit (2001). Modern actuarial risk theory, Dordrecht: Kluwer Academic Publishers.

[17] Schmeidler, D. (1989). "Subjective probability and expected utility without additivity," Econometrica 57, 571-587.

[18] Van Heerwaarden, A.E., R. Kaas \& M.J. Goovaerts (1989). "Properties of the Esscher premium calculation principle," Insurance: Mathematics and Economics 8, 261-267.

[19] Wu, X. \& J. Wang (2003). "On characterization of distortion premium principle," Astin Bulletin 33, 1-10.

[20] Yaari, M.E. (1987). "The dual theory of choice under risk," Econometrica 55, 95-115. 